\title{
Correction to: Student Next Assignment Submission Prediction Using a Machine Learning Approach
}

\author{
Y. K. Salal, M. Hussain, and P. Theodorou
}

\section{Correction to:}

Chapter "Student Next Assignment Submission Prediction Using a Machine Learning Approach" in:

A. A. Radionov and V. R. Gasiyarov (Eds.): Advances in Automation II, LNEE 729, https://doi.org/10.1007/978-3-030-71119-1_38

In the original version of the book, the following belated correction has been incorporated:

The author's last name has been changed from T. Paraskevi to P. Theodorou in Chapter 38. The book and the chapter have been updated with the change. 